\title{
KRAS NP_004976.2:p.K117X
}

National Cancer Institute

\section{Source}

National Cancer Institute. KRAS NP 004976.2:p.K117X. NCI Thesaurus. Code C147576.

A change in the amino acid residue at position 117 in the GT Pase KRas protein where lysine has been replaced by another amino acid. 